\title{
Pseudo-von Willebrand disease
}

INSERM

\section{Source}

INSERM. (1999). Orphanet: an online rare disease and orphan drug data base. Pseudovon Willebrand disease. ORPHA:52530

Platelet type Von Willebrand disease (PT-VWD) is a bleeding disorder characterized by mild to moderate mucocutaneous bleeding, which becomes more pronounced during pregnancy or following ingestion of drugs that have anti-platelet activity. PT-VWD is due to hyperresponsive platelets, resulting in thrombocytopenia. 